\title{
Comparative analysis of integument transcriptomes identifies genes that participate in marking pattern formation in three allelic mutants of silkworm, Bombyx mori
}

\author{
Xin Ding ${ }^{1} \cdot$ Junxia Liu ${ }^{1} \cdot$ Xiaoling Tong ${ }^{1} \cdot$ Songyuan $\mathrm{Wu}^{1} \cdot$ Chunlin $\mathrm{Li}^{1} \cdot$ Jiangbo Song ${ }^{1} \cdot$ Hai Hu ${ }^{1} \cdot$ Duan $\operatorname{Tan}^{1}$. \\ Fangyin Dai ${ }^{1}$
}

Received: 19 November 2018 / Revised: 4 August 2019 / Accepted: 8 August 2019/Published online: 2 September 2019

(C) The Author(s) 2019

\begin{abstract}
The diversity markings and pigment patterns in insects are outcomes of adaptive evolution. The elucidation of the molecular mechanism underlying variations in pigment patterns may improve our understanding of the origin and evolution of these spectacular diverse phenotypes. Melanin, ommochrome, and pteridine are the three main types of insect pigments, and the genes that directly participate in pigment biosynthesis have been extensively studied. However, available information on gene interactions and the whole pigment regulatory network is limited. In this study, we performed integument transcriptome sequencing to analyze three larval marking allelic mutants, namely, multi lunar $(L), L^{C}$, and $L^{C a}$, which have similar twin-spot markings on the dorsal side of multiple segments. Further analysis identified 336 differentially expressed genes (DEGs) between $L$ and Dazao (wild type which exhibits normal markings), 68 DEGs between $L^{C} /+$ and $+{ }^{L C} /++C$, and 188 DEGs between $L^{C a} /+$ and $+{ }^{L C a} /+{ }^{L C a}$. Gene Ontology (GO) analysis indicated a significant DEG enrichment of the functional terms catalytic activity, binding, metabolic process, and cellular process. Furthermore, three mutants share six common enriched KEGG pathways. We finally identified eight common DEGs among three pairwise comparisons, including Krueppel-like factor, TATA-binding protein, protein patched, UDP-glycosyltransferase, an unknown secreted protein, and three cuticular proteins. Microarray-based gene expression analysis revealed that the eight genes are upregulated during molting, which coincides with marking formation, and are significantly differentially expressed between marking and non-marking regions. The results suggest that the eight common genes are involved in the construction of the multiple twin-spot marking patterns in the three mutants.
\end{abstract}

Keywords Bombyx mori $\cdot$ Allelic mutants $\cdot$ Integument $\cdot$ Transcriptome $\cdot$ Pigment $\cdot$ Marking pattern

\section{Introduction}

Insects are the most diverse and abundant group, and more than 1 million species are distributed around the world. Their success relies on the variety of traits developed during their evolution, including the formation of various markings and

Electronic supplementary material The online version of this article (https://doi.org/10.1007/s10142-019-00708-w) contains supplementary material, which is available to authorized users.

Fangyin Dai

fydai@swu.edu.cn

1 State Key Laboratory of Silkworm Genome Biology, Key Laboratory of Sericultural Biology and Genetic Breeding, Ministry of Agriculture and Rural Affairs, College of Biotechnology, Southwest University, Chongqing 400715, China pigment patterns. The easily observable intra- and interspecific highly diversified body color patterns of insects are good system to studying the genetic mechanism of phenotypic evolution. In addition, diversified body color patterns are benefit for insects, such as avoid predators, mate choice, immunity, thermoregulation, and UV protection (Kronforst et al. 2012; Takahashi 2013; Wittkopp and Beldade 2009; Wittkopp et al. 2003). Therefore, it is essential to elucidate the molecular genetic mechanism of the occurrence and differentiation of insect coloring.

Melanin, ommochrome, and pteridine are the three major types of insect pigments. The genes involved in the pigments syntheses had been well identified in insects and are conserved in different species. However, in addition to the genes that directly participate in pigment synthesis, recent studies have shown that many regulatory genes are also involved in the regulation of the expression of pigment synthesis genes 
that determine coloring patterns. The Wnt family gene is revealed related to the establishment of color patterns of fruit fly, butterfly, and silkworm (Carroll et al. 1994; Gallant et al. 2014; Koshikawa et al. 2015; Martin et al. 2012; Martin and Reed 2014; Mazo-Vargas et al. 2017; Yamaguchi et al. 2013). The diversification of cis-regulatory elements of $w g$ (wingless) allows regulation of its expression, resulting in the spotting pattern in Drosophila guttifera and Bombyx mori (Koshikawa et al. 2015; Yamaguchi et al. 2013). $W n t A$, another member of the Wnt family, is also related to the establishment of black wing patterns in Heliconius genus through the diversification of cis-elements (Gallant et al. 2014; Martin et al. 2012; Martin and Reed 2014; MazoVargas et al. 2017). Except the Wnt genes, there are many other regulatory genes in different species. In Drosophila, optomotor-blind (omb), bric a brac (bab), abdominal-B (Abd-B), doublesex (dsx), Distal-less (Dll), and Engrailed (en) have been shown to regulate expression of pigment synthesis genes either directly or indirectly (Massey and Wittkopp 2016; Tong et al. 2014). In butterfly, Optix, Ubx, Antp, Dll, E75, spalt, and cortex genes have been shown that associated with the formation of eye spots on wings (Futahashi et al. 2012; Tong et al. 2014). In silkworm, upregulation of apontic-like gene and Toll signaling pathway receptor Spätzle3 promotes the pigmentation of silkworm larvae (KonDo et al. 2017; Yoda et al. 2014).

Identification of these genes and regulatory elements enriches our understanding of insect pigment regulatory mechanisms; it also reveals the complexity of the associated regulatory network. In addition, compared with the extensive studies that focus on the adult wing spot pigmentation of fruit flies and butterflies, investigations on the diversity of noticeable larval marking pigmentation remains limited (Shirataki et al. 2010). Over 100 years of genetic studies on the lepidopteran Bombyx mori have accumulated nearly 200 strains of mutants exhibiting variations in body color and stripes, thereby making it an ideal model for research on the molecular mechanism of complex color patterns in caterpillars. In silkworm, multi lunar $(L), L^{C}$, and $L^{C a}$ are three spontaneous mutations which have similar twin-spot markings on the dorsal multiple segments (Fig. 1); previous classical genetic analysis revealed that the three mutants were allelic mutations. Similar phenotypes and allele mutations suggest that these three mutants may have common regulatory basis. Fujiwara et al. revealed that Wnt1 gene was responsible for $L$ mutant by localized cloning (Yamaguchi et al. 2013). In this study, we performed RNA-seq of the three similar phenotypic mutants to identify more candidate genes that are involved in the formation of marking patterns of the $L$-type mutants, as well as provide a reference for understanding the genetic basis of markings formation in caterpillar.

\section{Results}

\section{Library construction and overview of RNA-seq}

The color pattern of silkworm larva is reformed at every molting period, prompting us to select the onset of molting (head capsule slippage, HCS) stage of the 4th molting for RNA-seq to analyze the expression levels of genes that are related to pigmentation. Three similar phenotypic mutants $(L$, $L^{C}$, and $L^{C a}$ ) were used in this study. The markings of the $L$ mutant are crescent in shape, whereas that of $L^{C}$ mutants are more triangulated and that of $L^{C a}$ are generally square with irregular edges. These similarities in phenotype suggest that the three mutants may have the same molecular basis in the formation of marking patterns. $L$ is a near isogenic line of wild-type Dazao, so we selected and used $L$ and Dazao for pairwise comparison. The homozygous embryo of $L^{C}$ and $L^{C a}$ is lethal, and twin-spot markings are observed only on the 5th and 8th segments in both $+{ }^{L C /+}+{ }^{L C}$ and $+{ }^{L C a} /+{ }^{L C a}$, which are generated by heterozygous $L^{C}$ and $L^{C a}$ inbred lines, respectively (Fig. 1). Thus, we selected $+{ }^{L C} /+{ }^{L C}$ and $+{ }^{L C a} /+{ }^{L C a}$ as controls of heterozygous $L^{C}$ and $L^{C a}$. Finally, a total of 18 transcriptome libraries containing three pairwise comparisons were constructed, including three mutants and the corresponding controls. Three biological duplicates of each sample were prepared and then sequenced on an Illumina HiSeq 2000 platform.

A total of 126.70-gigabase $(\mathrm{Gb})$ clean reads were generated, and the data size of each sample is approximately 6.23 to $9.04 \mathrm{~Gb}$, with a Q30 percentage (percentage of sequences with sequencing error rates $<1 \%$ ) varied between 92.75 and $93.38 \%$. The clean reads of each sample were mapped to the reference genome of the silkworm, and the mapped efficiency of the samples varied from 75.10 to $87.21 \%$ (Fig. 2). The obtained mapped reads were assembled and compared with the original annotation information of the reference genome, which identified a total of 1225 novel transcripts (Online Resource 1). The nucleotide sequences of new transcripts were blast in databases including NR, GO, Swiss-Prot, COG, KOG, KEGG, and Pfam. A total of 1111 new genes were finally annotated, and the number of new genes annotated in each database is shown in Table 1.

\section{Analysis of differentially expressed genes}

Based on the transcriptomes of three $L$-type multiple twin-spot marking pattern mutants and their control, we performed differentially expressed gene (DEG) analysis using edgeR (Robinson et al. 2010) in BMKcloud (http://www.biocloud. net) to identify the related genes involved in $L$-type marking pattern formation. Fragments per kilobase of transcript per million fragments mapped (FPKM) was used to calculate abundance of transcripts of each gene. Based on the 18 


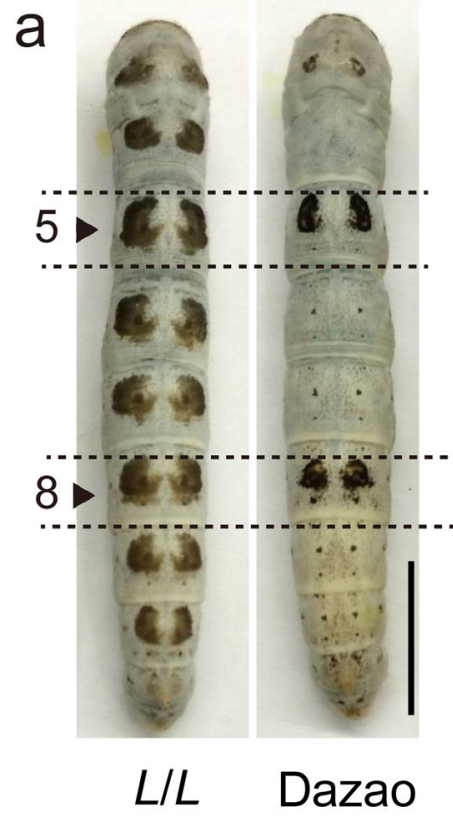

Fig. 1 The phenotype of $L$-type mutant and wild type at HCS of fourinstar larval stage. a Homozygous $L$ mutant and wild-type strain Dazao. $L$ is an inbred line of Dazao that was generated after several years of crossing. b Heterozygous $L^{C}$ mutant and $+{ }^{L c} /+{ }^{L c}$. The $L^{C}$ mutant possesses

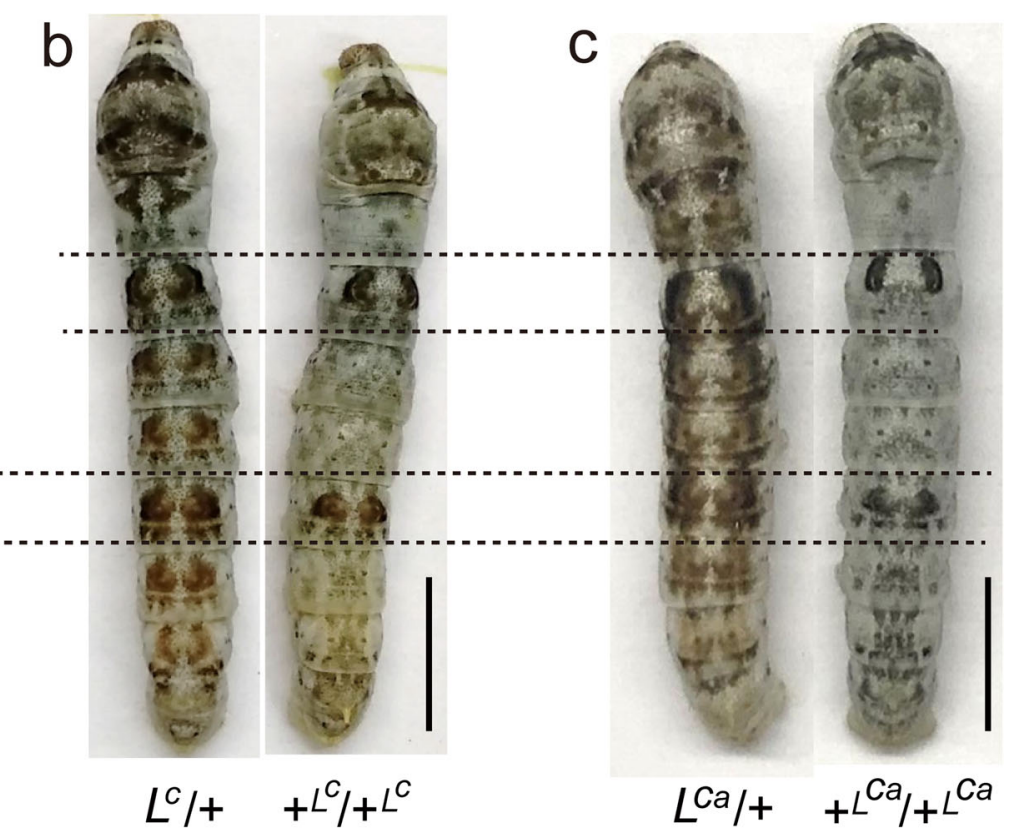

triangulated markings on the dorsal of multiple segments. $\mathbf{c}$ Heterozygous $L^{C a}$ mutant and the $+{ }^{L C a} /+{ }^{L C a}$. The $L^{C a}$ mutant possesses squared markings with irregular edge transcriptome libraries, a total of 12,095 expressed genes were identified and the FPKM values were calculated (Online Resource 2). Significant differential expression was considered to exist when the $\log _{2}$ fold change (logFC) of the mutant and control ratio is $>1$ at a threshold of $P$ value $<0.05$. After analysis, 336 significantly DEGs were detected between $L$ and Dazao, of which 189 were upregulated and 147 were downregulated; 68 significantly DEGs were detected between $L^{C} /+$ and $+{ }^{L C} /+{ }^{L C}$, which consisted of 51 upregulated and 17 downregulated genes; and 188 DEGs were identified between $L^{\mathrm{Ca}} /+$ and $+{ }^{L C a} /+{ }^{L C a}$, comprising 120 upregulated and 68 downregulated genes (Table 2 and Online Resource 2).

To validate our RNA-seq data, 12 DEGs were selected based on their up- and downregulated fold change values and assessed by RT-qPCR analysis. To intuitively compare RNA-seq data and RT-qPCR data, the gene expression levels generated by RT-qPCR were changed to $\log _{2}$ (fold change), which were then used in RNA-seq data analysis. The fold changes of the selected genes coincided with our RNA-seq data, indicating the reliability of our RNA-seq results (Fig. 3).

\section{Gene Ontology (GO) and KEGG pathway enrichment analysis of DEGs}

To assess the molecular function of DEGs in the three mutantcontrol pairwise comparisons $\left(L / L \mathrm{VS}\right.$ Dazao, $L^{C} /+\mathrm{VS}$ $+{ }^{L C} /+{ }^{L C}, L^{C a} /+\mathrm{VS}+{ }^{L C a} /+{ }^{L C a}$ ), GO categories and KEGG pathway enrichment analysis were performed. GO analysis divided the DEGs into three functional groups: biological process, cellular component, and molecular function (Fig. 4). The most significantly enriched terms in the three mutants included catalytic activity, binding, metabolic process, and cellular process. Then, the DEGs were mapped to reference pathways in the KEGG database, and the significantly enriched KEGG terms are shown in Fig. 5. These results show that the three mutants share six common enriched KEGG pathways, including hedgehog signaling pathway, retinol metabolism, metabolism of xenobiotics by cytochrome P450, drug metabolism-cytochrome P450, drug metabolism-other enzymes, and insect hormone biosynthesis (red in Fig. 5). The hedgehog signaling pathway is related to body plan and segment determination (Carballo et al. 2018; NussleinVolhard and Wieschaus 1980). In butterfly, the hedgehog signaling pathway has been shown to play a role in the establishment of eyespot patterns (Keys et al. 1999; Tong et al. 2012).

\section{The common DEGs are related to the multiple twin-spot marking formation in the three mutants}

To identify major DEGs linked to multiple twin-spot marking formation in the three similar phenotype mutants, a Venn diagram was constructed (Fig. 6). The results show eight DEGs that are common among the three mutants (Table 3). Among these eight DEGs, BGIBMGA008464 (secreted protein), BGIBMGA013828 (TATA-binding protein), NewGene6331 (patched protein), BGIBMGA014622 (UDP-glycosyltransferase), $B G I B M G A 010500$ (cuticular protein), $B G I B M G A 006120$ (Krueppel-like factor), and 
Fig. 2 Overview of RNA-seq. a The numbers at the end of samples name represent biological replicates of every strain. Q30 represent the sequencing error rate $<0.1 \%$. G represents gigabase. $\mathbf{b}$

Comparison efficiency statistics. The blue column represents unique mapped reads, the red column indicated multiple mapped reads, and the green column shows the unmapped reads a

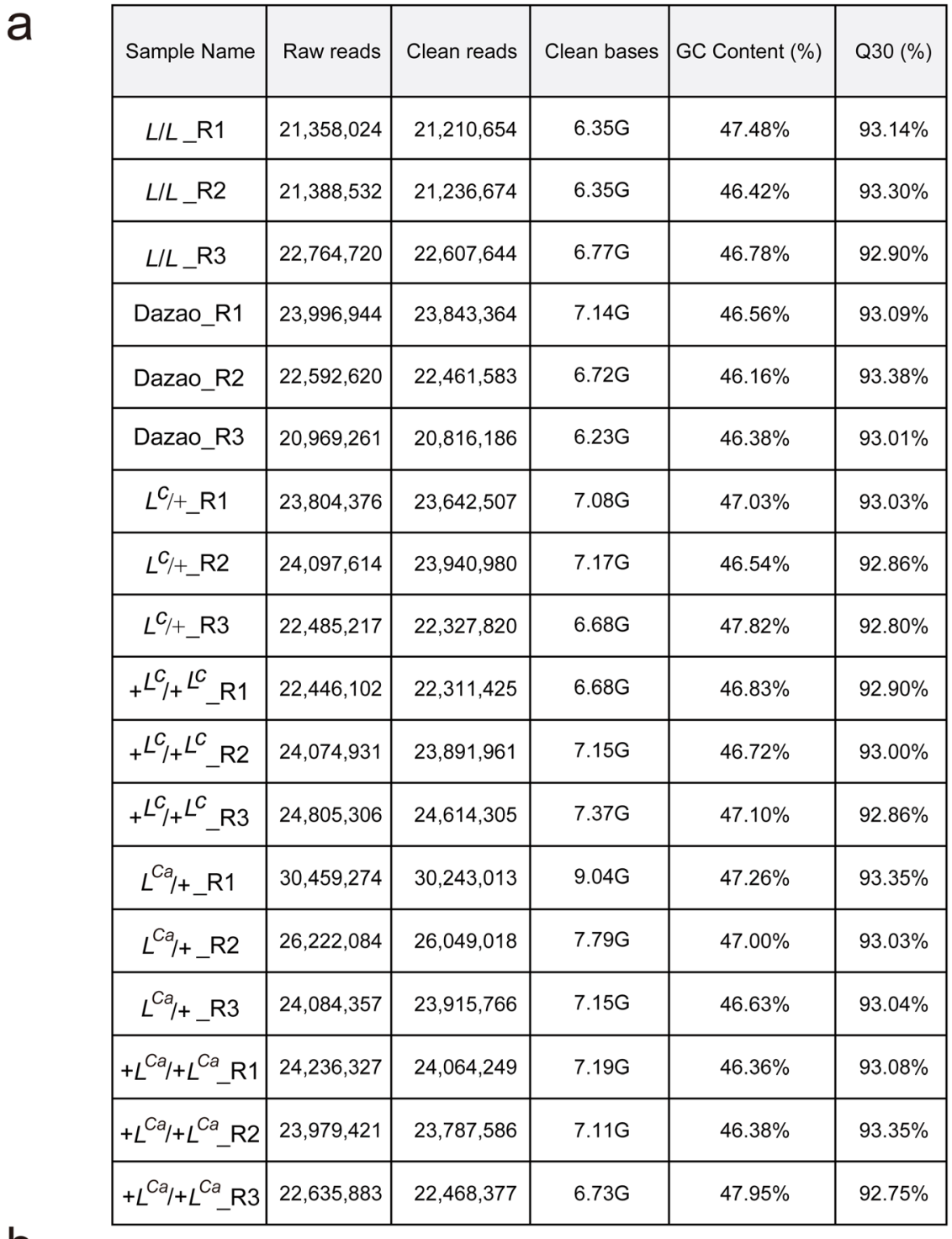

b

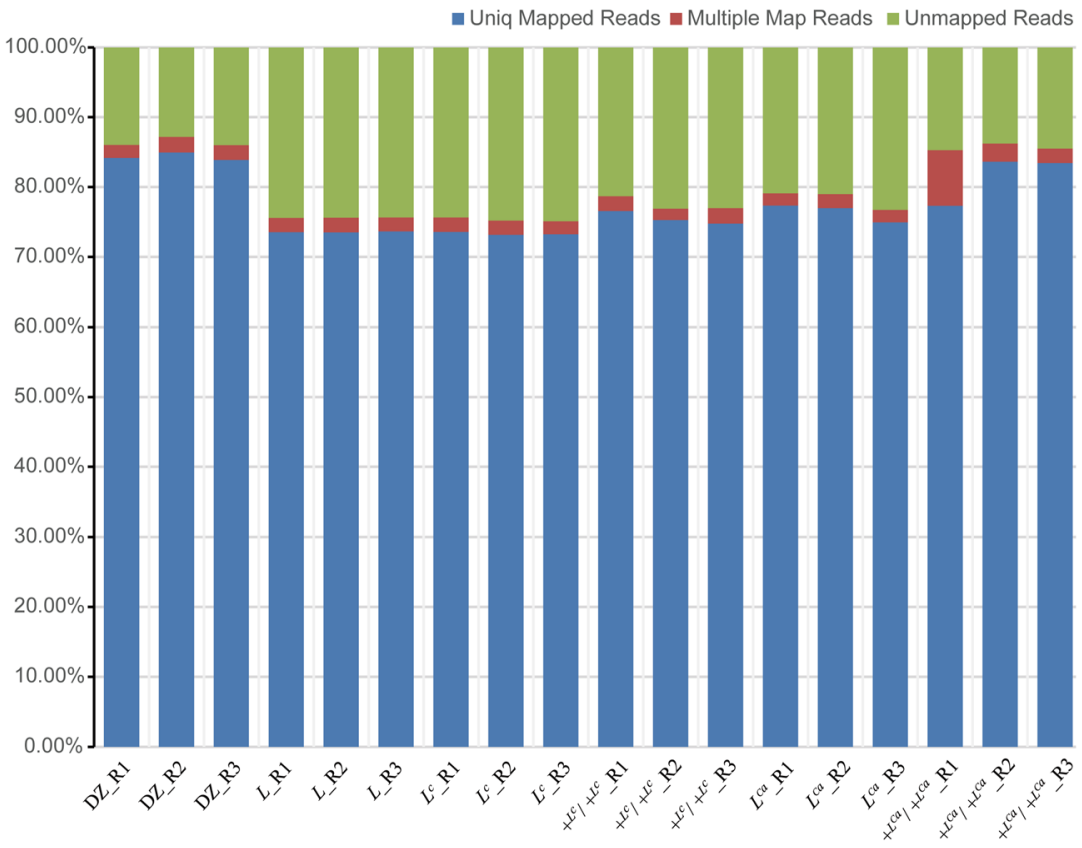


Table 1 Number of new genes annotated in databases

\begin{tabular}{ll}
\hline Annotated databases & New gene number \\
\hline COG & 189 \\
GO & 530 \\
KEGG & 307 \\
KOG & 534 \\
Pfam & 606 \\
Swiss-Prot & 458 \\
eggNOG & 995 \\
nr & 1082 \\
All & 1111 \\
\hline
\end{tabular}

NewGene_4929 (cuticular protein) are upregulated in the three mutants; BGIBMGA013915, which encodes a RR-2 motif cuticular protein, is downregulated in the three multiple twin-spot markings mutants.

To further analyze the relationship between the common genes and marking formation, we analyzed expression microarray data of the eight DEGs in the 4th instar period of the silkworm. Two of the 8 genes have no microarray data. The other 6 genes, BGIBMGA008464, BGIBMGA010500, BGIBMGA013828, BGIBMGA014622, BGIBMGA006120, and $B G I B M G A 013915$ were upregulated during molting, which coincides with marking formation (Fig. 7). The result indicates that the common genes may be involved in marking formation in the three mutants.

Then, we detected the expression levels of the eight genes in pigmented and non-pigmented regions of the three mutants. BGIBMGA010500, NewGene4929, BGIBMGA013828, NewGene6331, and BGIBMGA008464 are significantly upregulated in the pigment regions in all three mutants, $B G I B M G A 006120$ is only significantly upregulated in the pigmented regions of the $L^{C}$ mutant, BGIBMGA014622 is only significantly upregulated in the pigmented regions of the $L$ mutant, and BGIBMGA013915 is only significantly downregulated in the pigmented region of the $L^{C a}$ mutant (Fig. 8). These results further show that the identified common DEGs are closely related to mutant marking formation.

\section{Expression analysis of pigment genes}

The phenotype of the three $L$-type mutants $\left(L, L^{C}, L^{C a}\right)$ includes twin-spots on the dorsal side of multiple segments. To

Table 2 DEG analysis of the three pairwise comparisons

\begin{tabular}{llll}
\hline & Total DEGs & Upregulated & Downregulated \\
\hline$L / L$ VS Dazao & 336 & 189 & 147 \\
$L^{C /+} \mathrm{VS}+{ }^{L C} /+{ }^{L C}$ & 68 & 51 & 17 \\
$L^{C a} /+\mathrm{VS}+{ }^{L C a} /+{ }^{L C a}$ & 188 & 120 & 68 \\
\hline
\end{tabular}

investigate the basis of marking formation, we analyze the expression levels of major genes that participate in pigment synthesis. No significant change in expression levels was observed in most genes (Table 4). However, we performed RNA-seq of the integument of the mutants in HCS, whereas the expression of several major melanin synthesis-related genes expression is relatively very low. Previous studies in Papilio xuthus have shown that $P A H$ is expressed throughout molting; yellow and laccass 2 are expressed in the middle of the molt stage; Dat1, TH, DDC, ebony, tan, and black are expressed later until ecdysis (Futahashi et al. 2010; Futahashi and Fujiwara 2005). Therefore, we detected the expression levels of PAH, yellow, laccass 2, Dat1, TH, ebony, tan, and black in the integument of the mutants at $18 \mathrm{~h}$ after HCS through RT-qPCR (Fig. 9). In the three mutants, most of these genes are upregulated, indicating that melanin synthesis is aggravated, which coincides with the multi-markings phenotype. The selected stage to perform transcriptome analysis is HCS of the 4th instar, which occurs prior to melanin biosynthesis and marking formation. These findings suggest that the identified common DEGs are upstream regulators of pigment synthesis-related genes.

\section{Discussion}

\section{L-type mutants are complex pigment mutants containing melanin and ommochrome}

Our analysis revealed that the genes that participate in melanin synthesis are upregulated in mutants at $18 \mathrm{~h}$ after HCS, suggesting that melanin synthesis pathway may be activated (Fig. 9). In addition, we also found that Bmcardinal, which encodes a phenoxazinone synthetase that participates ommochrome synthesis (Osanai-Futahashi et al. 2016), is significantly upregulated in the $L$ and $L^{C a}$ mutants (Table 4). Ommochrome is a group of insect pigments that mainly exist in insect eyes, eggs, and wings. It has also been reported that ommochrome are also produced in the epidermis of insects such as Gryllus bimaculatus, Carausius morosus, and Mantis religiosa (Linzen 1974). However, compared with the widely studied synthesis mechanism of epidermal melanin, the function and synthesis mechanism of ommochrome in the epidermis of insect larvae remains unclear. Studies have revealed that quail $(q)$ and quail-like $\left(q-l^{P}\right)$ are complex pigment mutants that contain melanin, ommochrome, and pteridine, and Bmcardinal is also upregulated in the $q$ and $q-l^{P}$ mutants (Nie et al. 2014; Wang et al. 2017). Moreover, the absence of Bmcardinal activity results in suppressed red pigmentation around the eyespots of larval epidermis in the $p^{S}$ strain (Osanai-Futahashi et al. 2016). Although Bmcardinal is upregulated in $q$ and $q-l^{P}$, the two mutants have no distinct eyespots and red pigmentation, and 


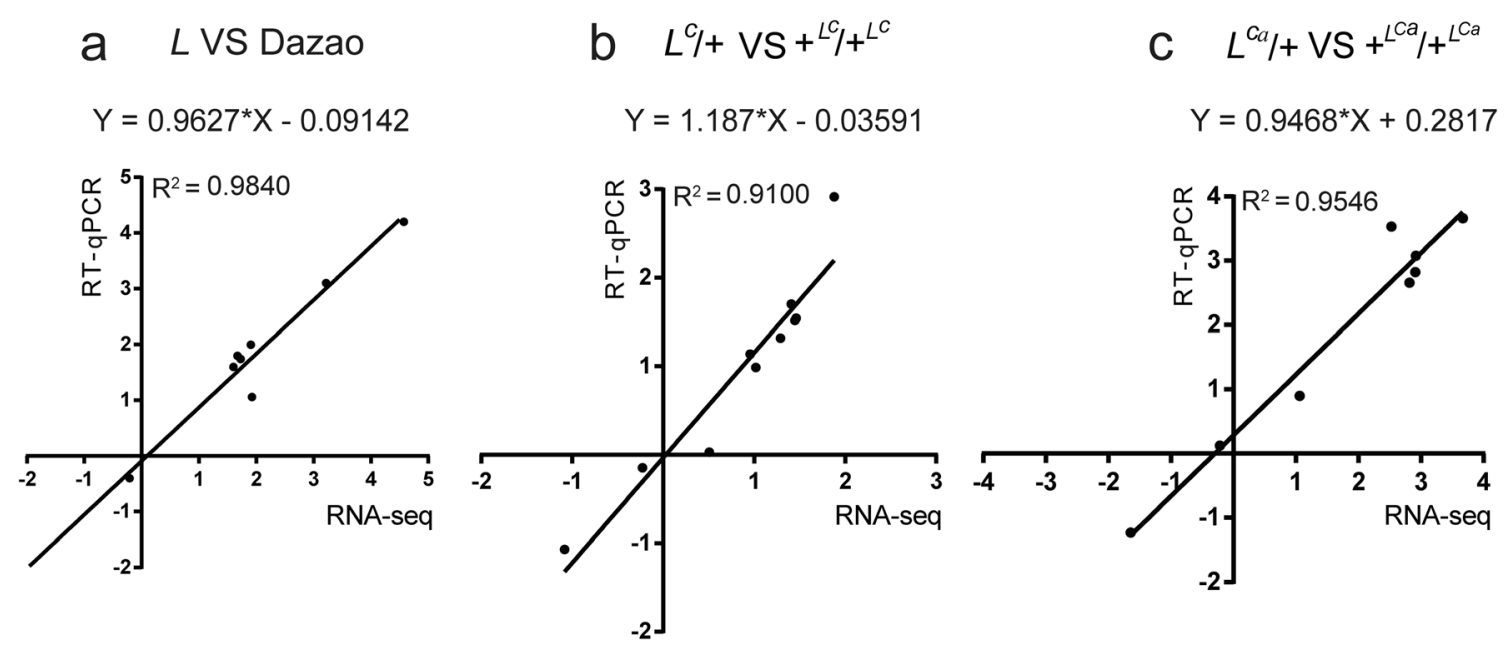

Fig. 3 Comparison of gene expression values obtained by RNA-seq and real-time fluorescence quantitative PCR (RT-qPCR). The $x$-axis shows the $\log _{2} \mathrm{FC}$ from RNA-seq, the $y$-axis depicts the same values from RTqPCR using the $2^{-\Delta \Delta \mathrm{c}(\mathrm{t})}$ algorithm. The $R^{2}$ values show the correlation

we infer that the upregulated Bmcardinal in $q, q-l^{P}, L, L^{C}$, and $L^{C a}$ results in different background skin colors compared with the wild type. In summary, the $L$-type mutants are complex ratio between RNA-seq and RT-qPCR. a The $\log \mathrm{RT}_{2} \mathrm{FC}$ of $L$ mutant comparison of the wild-type Dazao. b The $\log _{2} \mathrm{FC}$ of heterozygous $L^{C}$ mutant and the wild-type $+{ }^{L C} /+{ }^{L C}$. c The $\log _{2} \mathrm{FC}$ of heterozygous $L^{C a}$ mutant and the wild-type $+{ }^{\mathrm{LCa} /+}{ }^{\mathrm{LCa}}$

pigment mutants that contain melanin and ommochrome and may be utilized in studying ommochrome pigments in insect larval epidermis.

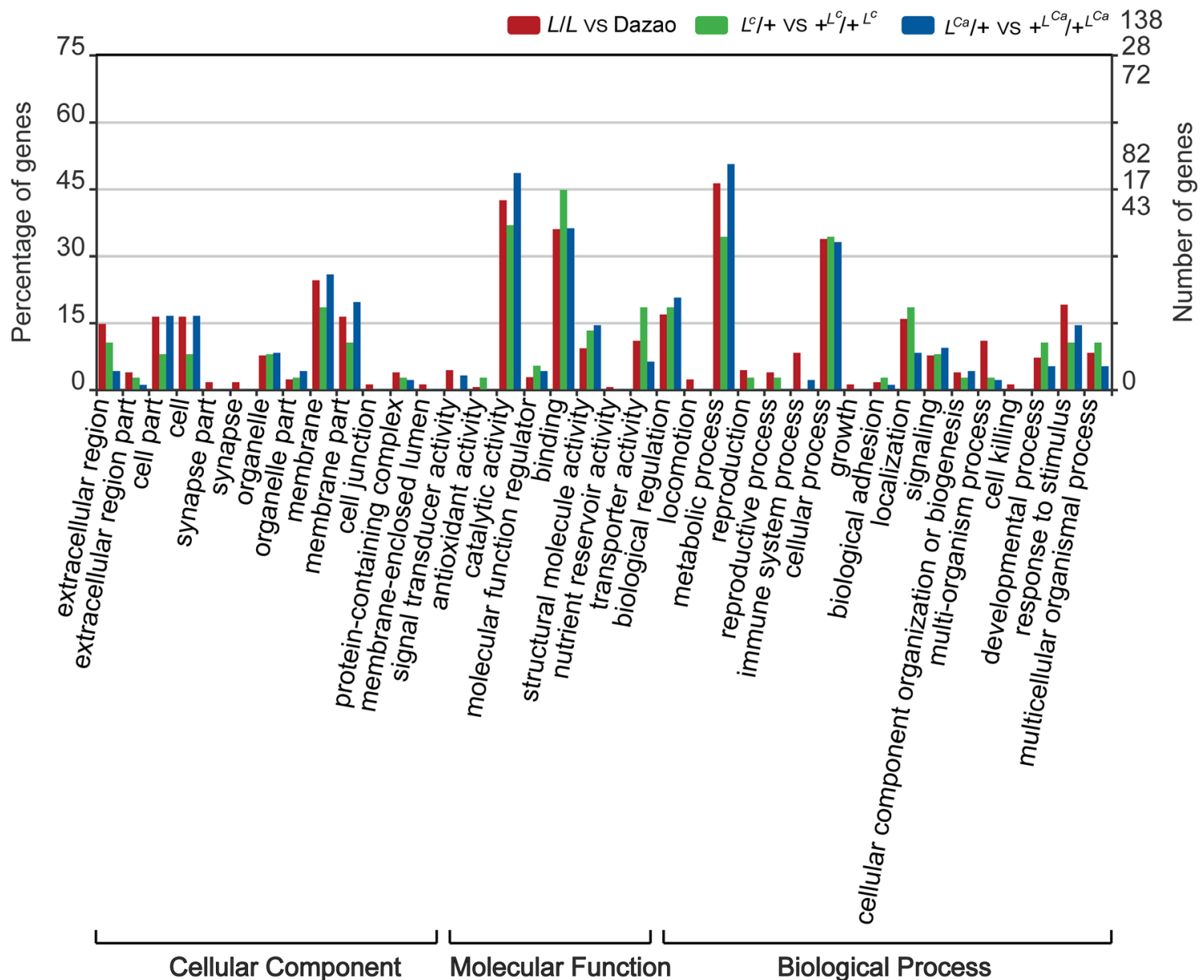

Fig. 4 GO analysis of DEGs in the three pairwise comparisons. The annotated DEGs were classified into the functional categories of cellular component, molecular function, and biological process. Various colors represent different pairwise comparisons 
Fig. 5 KEGG pathway enrichment analysis of DEGs in the three pairwise comparisons. a $L / L$ VS Dazao. b $L^{C} /+$ VS $+{ }^{L C} /+{ }^{L C}$. c $L^{C a} /+\mathrm{VS}+{ }^{L C a} /+{ }^{L C a}$. The $x$-axis indicates the enrichment factor. The $y$-axis shows different pathways a

Statistics of Pathway Enrichment

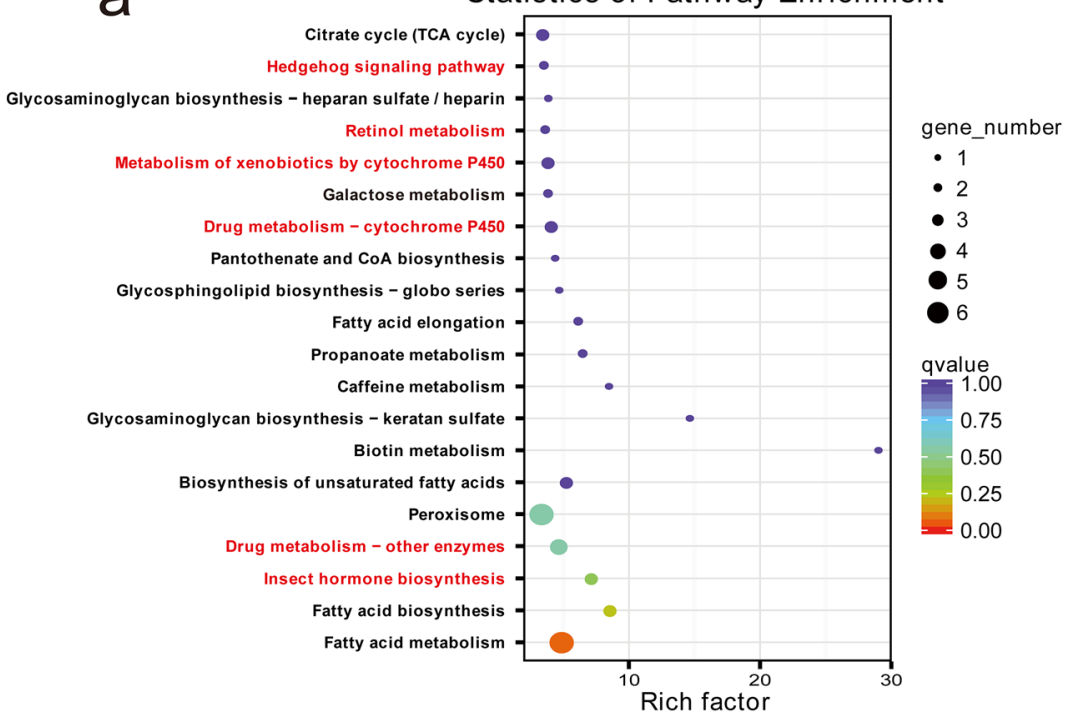

b

Statistics of Pathway Enrichment

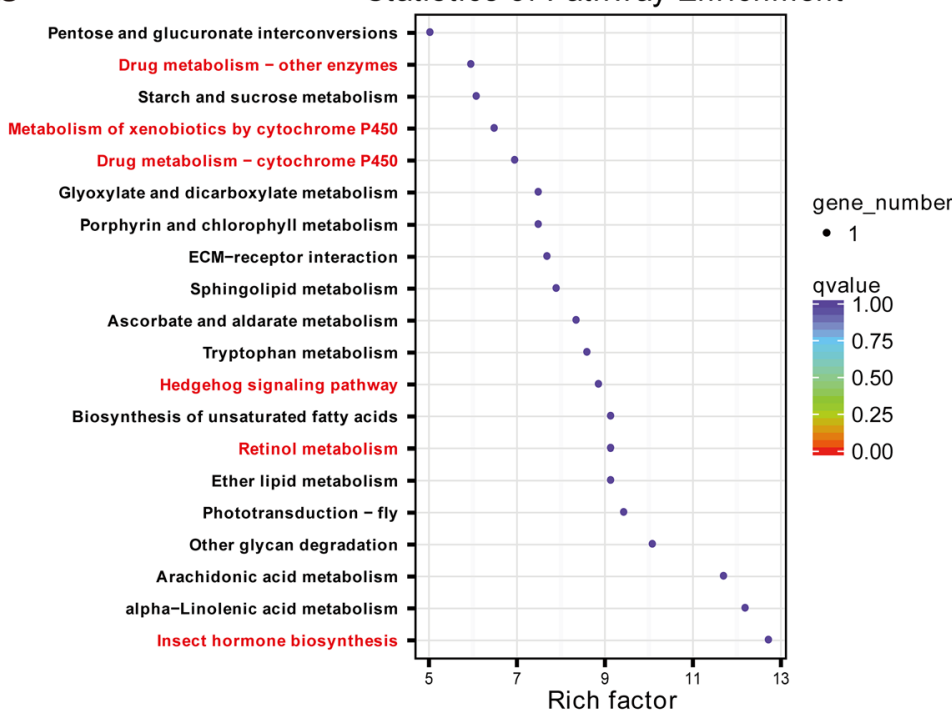

C

Statistics of Pathway Enrichment

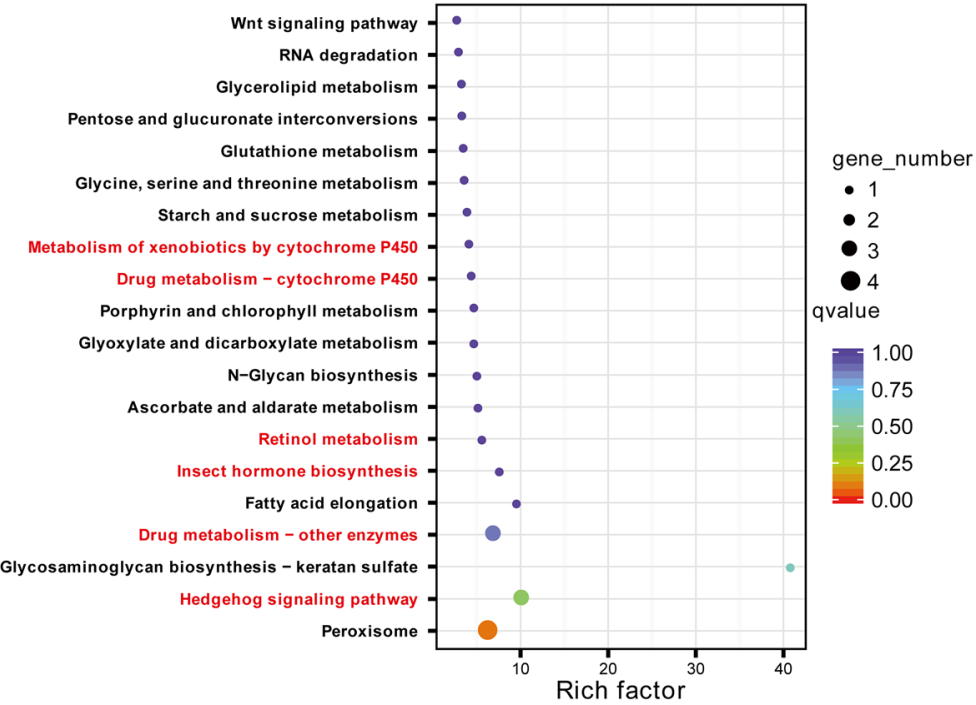




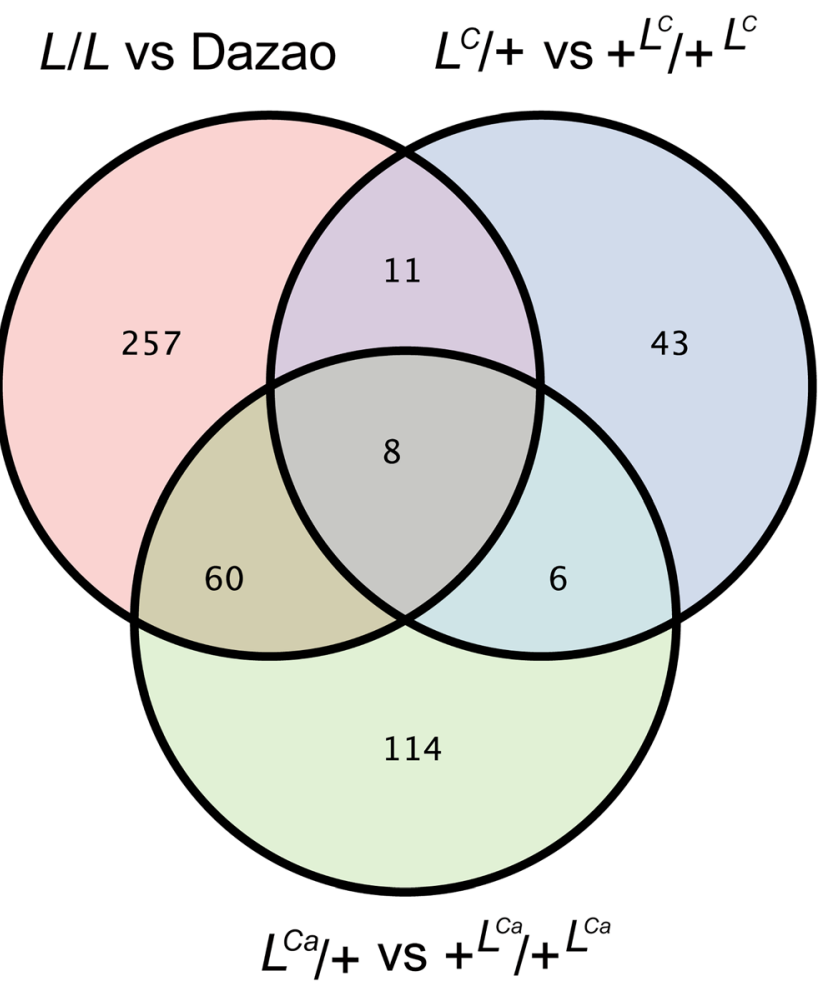

Fig. 6 Venn diagram of DEGs. The eight overlapping genes are common to the three pairwise comparisons. The numbers 257,43 , and 114 represent the specific number of DEGs detected in the $L, L^{C}$, and $L^{C a}$ mutants

\section{The identified DEGs are related to pigmentation in the three mutants}

Except for the analysis of genes that are directly involved in pigment synthesis, we hope to identify novel genes that are relate to pigment synthesis and the establishment of the marking patterns in the three mutants via transcriptional analysis. We identified eight common DEGs in the three mutants. One of these is UDP-glycosyltransferase, which paralogous to the $G b$ locus (Daimon et al. 2010). Loss of UDPglycosyltransferase in white cocoons prevents glycosylation of the 5-O position of dietary quercetin, thereby resulting in a significant decrease in total flavonoid content. To date, no study has on the role of UDP-glycosyltransferase in insect epidermal pigment synthesis has been conducted. This gene has also been identified in the butterfly wing color pattern transcriptome, suggesting that it might be involved in melanin formation (Zhang et al. 2017). In addition, some studies have shown that UDP-glycosyltransferase is involved in the formation of insect epidermis and tyrosine metabolism, and tyrosine is essential to melanin synthesis. We hypothesize that UDPglycosyltransferase alters the state of melanin and/or melanin precursor substances and participate in the transport of these substances through glycosylation.

Fujiwara previously identified the $L$ gene by positional cloning and reported that periodic expression of wnt 1 in response to ecdysteroid generates twin-spot markings of $L$ (Yamaguchi et al. 2013). However, we note that the Wnt1 and Wnt pathway genes were not included in the list of DEGs; the possible reasons is that the stage we performed RNA-seq is HCS, which is later than the stage of the upregulation of Wnt1 gene in $L$ mutant, and that we could not detect the differentially expressed between WT and the mutants. The other reason may be that Wnt 1 regulates body color formation in other ways which is independent of the Wnt signaling pathways, such as interaction with other signaling pathways. Previous studies have shown that the Wnt and Hedgehog signaling pathways both play roles in cell proliferation, differentiation, and embryo patterning in Drosophila, and the two signaling pathways have been proven to be not isolated. Some regulators have been identified to have the same effect on the Wnt and Hedgehog pathways, and crosstalk analysis identified hundreds of common proteins between the two pathways (Swarup et al. 2015; Toku et al. 2011). In this study, the patched gene (Table 3), a receptor of Hedgehog signaling, was significantly upregulated in the three mutants, suggesting that Hedgehog signaling participates in mutant phenotype construction. Network crosstalk analysis has indicate that the patched gene may be a node of the Wnt and Hedgehog signaling crosstalk networks (Toku et al. 2011). However, although studies involving butterfly revealed that the two pathways also participate in eyespots patterning (Keys et al. 1999; Tong et al. 2012), no study has shown that these interact with each other during this particular process. Our study suggests that the $L$ mutant is an ideal material for investigation two
Table 3 Common DEGs in the three mutants

\begin{tabular}{lllll}
\hline Gene & Annotation & $L / L$ VS Dazao & $L^{C} /+\mathrm{VS}+{ }^{L c} /+{ }^{L c}$ & $L^{C a} /+\mathrm{VS}+{ }^{L c a} /+{ }^{L c a}$ \\
\hline BGIBMGA010500 & Cuticular protein & $\mathrm{Up}$ & $\mathrm{Up}$ & $\mathrm{Up}$ \\
NewGene4929 & Cuticular protein & $\mathrm{Up}$ & $\mathrm{Up}$ & $\mathrm{Up}$ \\
BGIBMGA006120 & Krueppel-like factor & $\mathrm{Up}$ & $\mathrm{Up}$ & $\mathrm{Up}$ \\
BGIBMGA013828 & TATA-binding protein & $\mathrm{Up}$ & $\mathrm{Up}$ & $\mathrm{Up}$ \\
NewGene6331 & Patched protein & $\mathrm{Up}$ & $\mathrm{Up}$ & $\mathrm{Up}$ \\
BGIBMGA014622 & UDP-glycosyltransferase & $\mathrm{Up}$ & $\mathrm{Up}$ & $\mathrm{Up}$ \\
BGIBMGA008464 & Unknown secreted protein & $\mathrm{Up}$ & $\mathrm{Up}$ & $\mathrm{Up}$ \\
BGIBMGA013915 & RR2 cuticular protein & Down & Down & Down
\end{tabular}




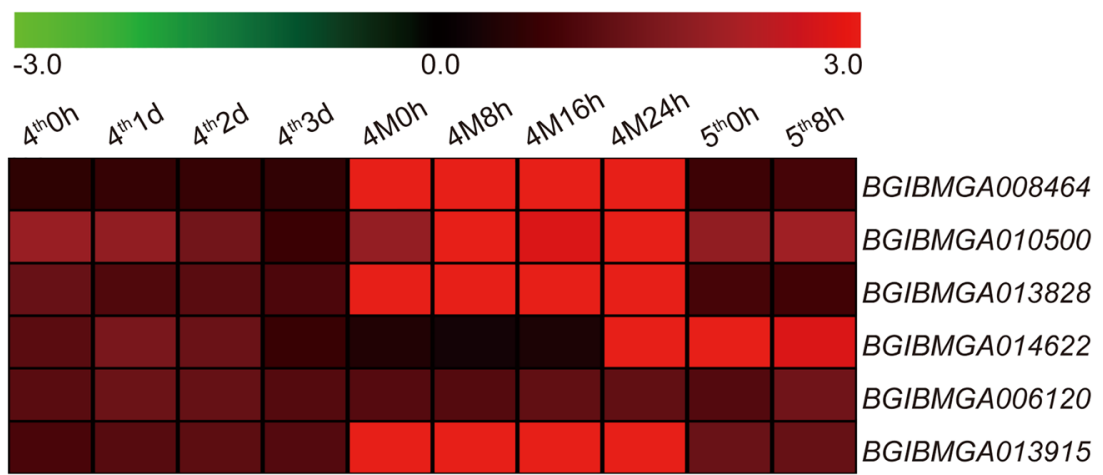

Fig. 7 Expression microarray data analysis of the common DEGs in the 4th instar period of the silkworm. The color bar represents gene expression levels, red means high expression, and green represents low expression. The columns represent 10 stages from 4 th instar to 5 th instar. 4 th $0 \mathrm{~h}$, the start of 4 th instar; 4th $1 \mathrm{~d}$, the first day of 4th instar; 4th2d, the

important pathways and their interactions in relation to color pattern formation in insects.

There are three cuticular proteins in the eight common DEGs, which include two upregulated (BGIBMGA010500 and NewGene_4929) and one downregulated (BGIBMGA013915) gene. The two genes, namely, BGIBMGA010500 and NewGene_4929, may be the common substances for color-pattern formation in three mutants. Cuticular proteins constitute the main components of insect epidermis. Body color of insects depends on the content of pigment substances in the epidermis, and normal epidermis construction serves as basis for establishing body color second day of 4th instar; 4th3d, the third day of 4th instar; 4M0h, start of 4th molting; 4M8h, the 8th hour of 4th molting; 4M16h, the 16th hour of 4th molting; 4M24h, the 24th hour of 4th molting; 5 th0h, the start of 5 th instar; 5 th $8 \mathrm{~h}$, the 8 th hour of the 5 th instar

pattern. Dai et al. found that the lack of a low-complexity epidermal protein BmorCPH24 in silkworm causes abnormal markings, which suggests that coloration in silkworm larvae is influenced by cuticular proteins (Xiong et al. 2017). Previous transcriptomes analyses have been identified a number of cuticular proteins in the pigmented integument of silkworm strains, including quail (q) (Nie et al. 2014), quail-like ( $q-l^{p}$ ) (Wang et al. 2017), black dilute (bd) (Wu et al. 2016), sexcontrolled melanism ( $\mathrm{sml}$ ) (He et al. 2016), and the epidermis of larvae and wings of adult in butterfly (Zhang et al. 2017), suggesting that cuticular proteins are important for epidermal construction and pigmentation. However, the identified
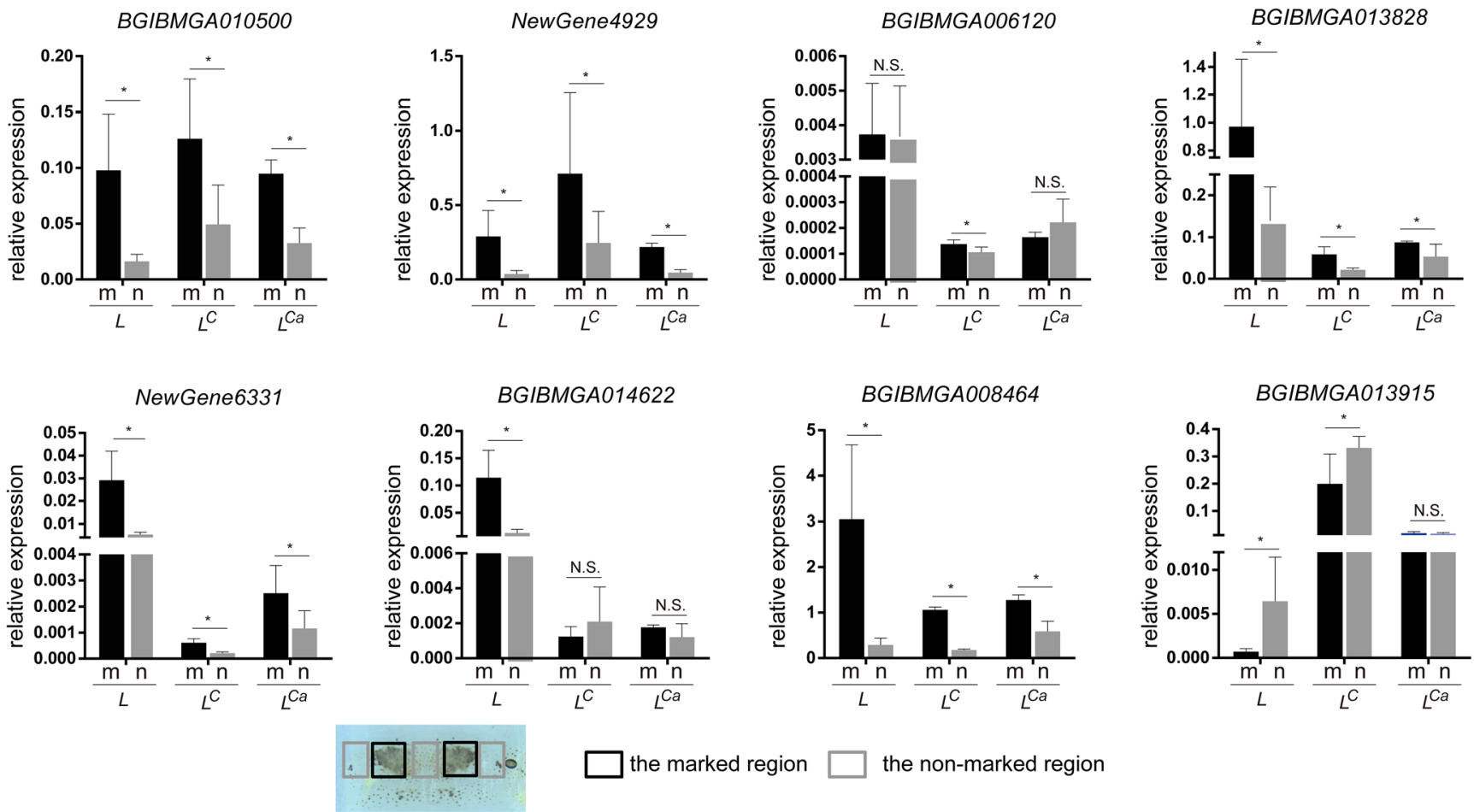

the marked region

the non-marked region

Fig. 8 The eight common DEGs specifically expressed in the marking region of the three mutants. $\mathrm{m}$, marking region; $\mathrm{n}$, non-marking region. $* P<0.05$, $n=3$ 
Table 4 Analysis of pigment biosynthesis genes expression in RNA-seq

\begin{tabular}{|c|c|c|c|c|}
\hline \multirow[t]{2}{*}{ Gene ID } & \multirow[t]{2}{*}{ Gene name } & \multicolumn{3}{|c|}{ Fold change* } \\
\hline & & $L \mathrm{VS} \mathrm{DZ}$ & $L^{C} \mathrm{VS}+{ }^{L C} /+^{L C}$ & $L^{C a} \mathrm{VS}+{ }^{L C a} /+{ }^{L C a}$ \\
\hline \multicolumn{5}{|c|}{ Key genes for biosynthesis of melanin } \\
\hline BGIBMGA000563 & $T H$ & -0.9457 & -0.172695 & 0.652021 \\
\hline BGIBMGA003199 & $D d c$ & 0.500719 & 0.5696452 & -0.39646 \\
\hline BGIBMGA003866 & $P A H$ & 0.331012 & 0.1114096 & 0.422383 \\
\hline BGIBMGA008538 & iAANAT & 0.022435 & $-4.15 \mathrm{E}-05$ & -0.08031 \\
\hline BGIBMGA012088 & black & - & - & - \\
\hline BGIBMGA006740 & laccase 2 & 1.659908 & 0.4407536 & 2.150171 \\
\hline BGIBMGA000031 & ebony & 0.41412 & -0.063488 & -0.06704 \\
\hline BGIBMGA002077 & $\tan$ & -0.26242 & -0.052286 & -0.13387 \\
\hline BGIBMGA001149 & yellow & 0.595242 & -0.240408 & -1.85824 \\
\hline BGIBMGA014032 & yellow- $f 2$ & -1.36087 & -0.977823 & -0.96286 \\
\hline BGIBMGA007253 & yellow-e & -0.34174 & -0.127416 & -0.02174 \\
\hline BGIBMGA003918 & yellow-f4-2 & -0.48026 & -0.725304 & -0.12375 \\
\hline BGIBMGA007254 & yellow-d & -0.64256 & 0.1928174 & -0.37524 \\
\hline BGIBMGA007255 & yellow- $h 2$ & -0.10514 & 0.2480949 & 0.363732 \\
\hline BGIBMGA014224 & yellow $x$ & 0.006538 & -0.472216 & -0.09412 \\
\hline BGIBMGA010917 & yellow-f4 & -0.35039 & 0.0255857 & -0.80856 \\
\hline \multicolumn{5}{|c|}{ Key genes for biosynthesis of ommochrome } \\
\hline BGIBMGA006740 & Bmcardinal & 1.659908 & 0.4407536 & 2.150171 \\
\hline BGIBMGA007285 & $B m G C-1$ & -0.2748 & 0.5931566 & 0.241481 \\
\hline BGIBMGA007286 & $B m G C-2$ & -0.18631 & 0.2416701 & -0.24799 \\
\hline BGIBMGA007856 & Kynurenine formamidase & 0.767844 & -0.044082 & 0.700758 \\
\hline \multicolumn{5}{|c|}{ Key genes for biosynthesis of pteridine } \\
\hline BGIBMGA001235 & GTP-cyclohydrolase-a & 0.230366 & -0.094524 & 0.105837 \\
\hline BGIBMGA008134 & GTP-cyclohydrolase-b & - & - & - \\
\hline BGIBMGA003643 & PTPS & -0.593526 & -0.069666 & 0.053928 \\
\hline
\end{tabular}

*The expression is signification upregulated when the value of fold change is $>1$ and signification downregulated when the value of fold change is $<-1$ cuticular proteins in these studies are largely different, although the same pigments occur in insects. We have two conjectures that may explain this discrepancy. (1) Various species employ different cuticular proteins that bind to the same pigment to form stable structures and/or (2) various developmental stages employ different cuticular proteins that interact with pigments. Further investigation on the underlying mechanism is thus warranted, which may also provide insights into the evolution of insects.

Except for the formation of the same marking pattern among the three mutants, fine regulation of different pigmentation ranges requires further investigation. According to the Venn diagram of DEGs (Fig. 6), there are 257 specific DEGs in $L, 43$ specific DEGs in $L^{C}$, and 114 specific DEGs in $L^{C a}$. We hypothesize that the specific DEGs may be related to the fine regulation of different pigmentation ranges among the three mutants. However, how the identified common DEGs participate in marking pattern formation among the three mutants and which genes are involved in the fine regulation of the different pigment ranges require further studies.

\section{Conclusions}

In this study, we analyzed the transcriptome of integuments of three allelic multiple twin-spot markings mutants and identified a number of DEGs, which were further subjected to GO and KEGG enrichment analyses. We observed that the genes involved in the biosynthesis of melanin and ommochrome were upregulated in the three mutants, suggesting their role in the generation of markings. We also identified common DEGs in the three mutants that may act as upstream regulators of pigment synthesis-related genes. The common DEGs are upregulated during molting, which coincide with marking formation and are significantly differentially expressed between marking and non-marking regions, suggesting that the 


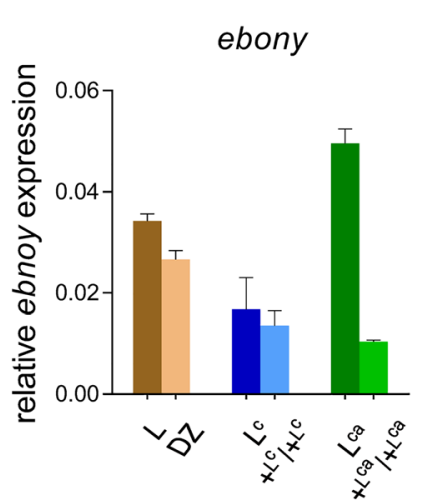

TH

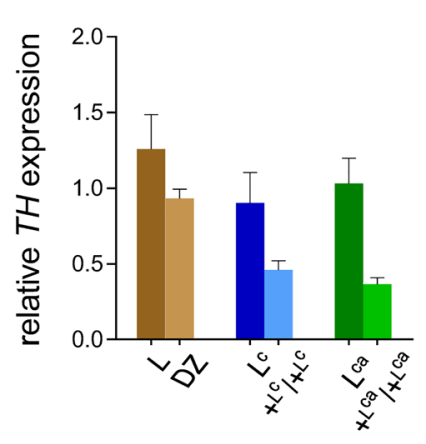

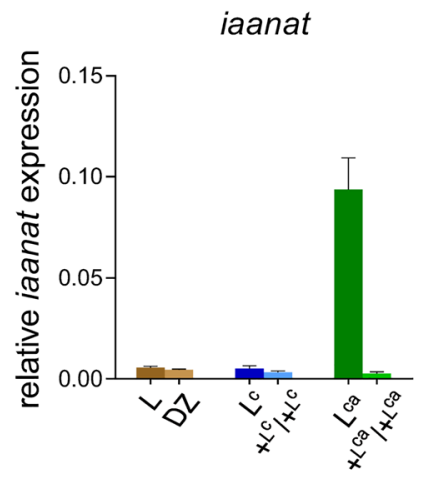

$\tan$
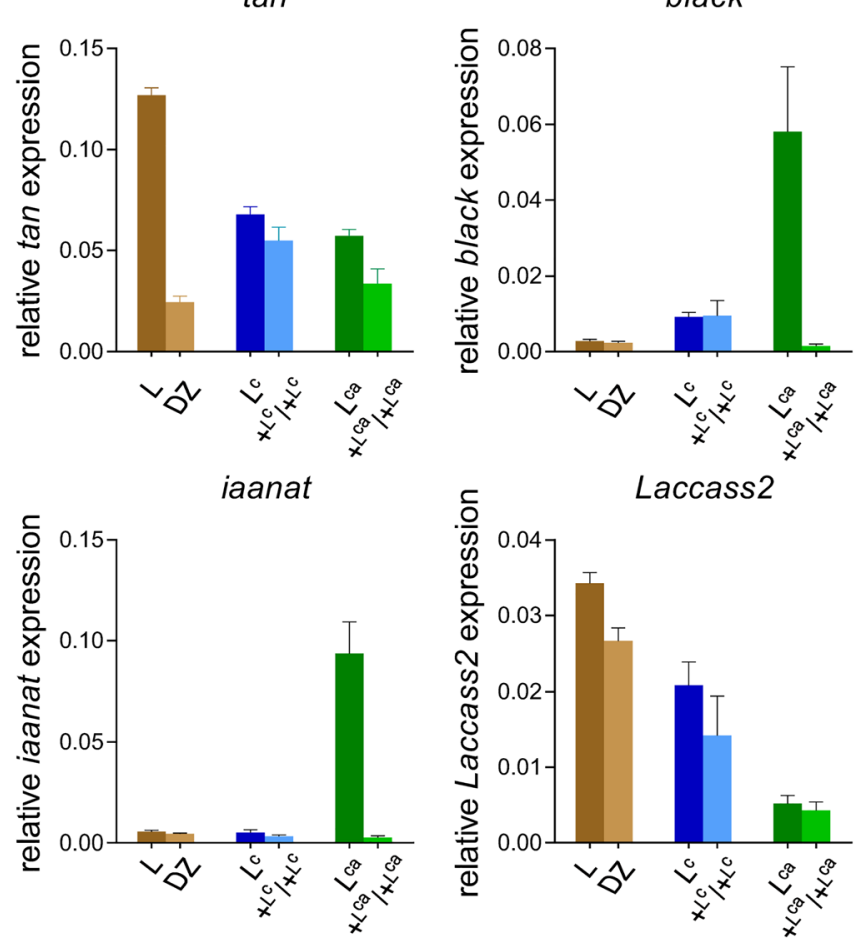

yellow

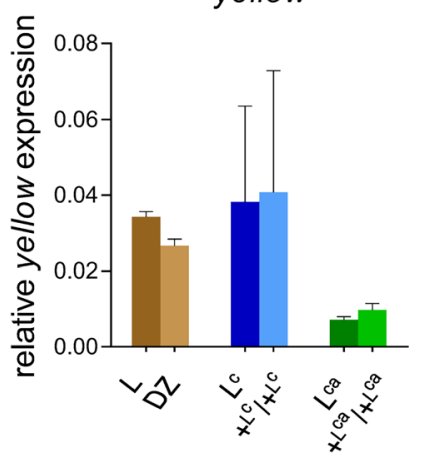

$P A H$

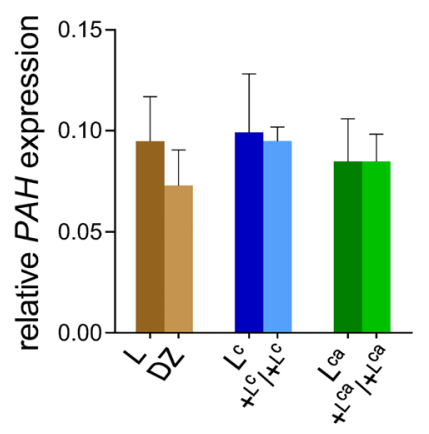

Fig. 9 RT-qPCR analysis of major melanin biosynthesis pathway genes in the three mutants. Various-colored columns represent different strains

common DEGs are involved in the formation of the $L$-type marking pattern. Except for the common DEGs, we have identified specific DEGs in the three mutants, which may be related to the fine regulation of the different pigmentation ranges in the three mutants. This research provides a reference for understanding of the genetic basis of $L$-type mutants.

\section{Materials and methods}

\section{Silkworm strains}

The silkworm strains used in this study were obtained from the Silkworm Gene Bank in Southwest University in Chongqing, China. The larvae were fed fresh mulberry leaves under standard conditions with $75 \%$ relative humidity at $25 \pm$ $2{ }^{\circ} \mathrm{C}$. $L$ and Dazao were used in a pairwise comparison; $L$ is an inbred line of the Dazao that was obtained after years of crossing. $L^{C} /+{ }^{L c}$ and $+{ }^{L c} /+{ }^{L c}$, and $L^{C a} /+{ }^{L C a}$ and $+{ }^{L C a} /+{ }^{L C a}$ were also used in pairwise comparisons.

\section{cDNA library preparation and Illumina RNA-seq}

The integuments were dissected from four individuals of each strain on HCS of the 4th instar; fat body and the trachea, which adhere to the integument, were removed using tweezers. The samples were stored at $-80^{\circ} \mathrm{C}$ in TRIzol reagent after quick freezing with liquid nitrogen. The dissections are conducted in a systematic manner between different groups to reduce experimental error. Total RNA of each mixture, which contains the pooled integuments of four individuals, was isolated using TRIzol reagent (Invitrogen, Carlsbad, CA, USA) according to manufacturer's instructions. RNA purity and concentration were assessed using a NanoDrop ND-2000C spectrophotometer (Thermo, Waltham, MA, USA). RNA integrity was assessed using the Agilent Bioanalyzer 2100 system (Agilent Technologies, Palo Alto, CA, USA). Then, mRNA enriched and fragment interruption, cDNA synthesis, the addition of adapters, PCR amplification, and Illumina sequencing were performed by Beijing Biomarker Technologies (Beijing, China).

\section{Data analysis}

Raw reads from the Illumina HiSeq, which are based on the sequencing by synthesis (SBS) technology, were evaluated using the Phred formula (Ewing et al. 1998). Low-quality reads including $N$ with a ratio $>10 \%$ and the number of the bases $Q \leq 10$ that accounts for $>50 \%$ of the whole reads were ruled out to obtain high-quality clean data. Then, the clean data were mapped to the silkworm reference genome (ftp:// ftp.ensemblgenomes.org/pub/metazoa/release-37/fasta/ bombyx_mori/) using TopHat2 (Kim et al. 2013), which is based on the alignment software Bowtie2 (Langmead et al. 2009). Aligned efficiency (mapped reads accounts for the percentage of clean reads) was calculated to evaluate utilization 
of the transcriptome data. Cuffquant and Cuffnorm used FPKM (Florea et al. 2013) as indicator of the level of transcriptional or gene expression, with FPKM $=$ cDNA fragments/mapped fragments (millions) $\times$ transcript length $(\mathrm{kb})$. Differential expression analysis was performed based on the FPKM of genes with a threshold $P<0.05$. Functional annotations of DEGs were analyzed using BLAST (Altschul et al. 1997) and HMMER. GOseq and KOBAS2.0 were used to perform GO and KEGG enrichment analyses of differentially expressed transcripts, respectively.

\section{Identification and functional annotation of new genes}

Cufflinks software (Roberts et al. 2011) was used to assemble the mapped reads and compare with the reference genome annotation information to identify novel transcripts and genes. The transcripts without corresponding templates in the reference genome annotation information were defined as new transcripts and new genes. The new genes were blast in NR, GO, Swiss-Prot, COG, KOG, and KEGG through blastX. The amino acid sequences of the new genes were predicted and then blast in Pfam with HMMER [136] software to obtain annotation information of the new genes.

\section{RT-qPCR}

The primers used for RT-qPCR were selected from qPrimerDB (Lu et al. 2018). RT-qPCR was performed on a CFX96 ${ }^{\mathrm{TM}}$ Real-Time PCR Detection System (Bio-Rad, Hercules, CA USA) with a SYBR Green RT-qPCR Mix (Bio-Rad). The PCR conditions were as follows: $95{ }^{\circ} \mathrm{C}$ for 3 min followed by 40 cycles of $95^{\circ} \mathrm{C}$ for $10 \mathrm{~s}$ and $60^{\circ} \mathrm{C}$ for 30 s. Three biological repeats were performed for each genotype, and each sample was analyzed in triplicate to rule out technical errors. Relative expression was calculated using the $2^{-\Delta \Delta \mathrm{Ct}}$ method (Livak and Schmittgen 2001) with B. mori eukaryotic translation initiation factor $4 A$ as reference.

\section{Microarray analysis of spatiotemporal gene expression}

Based on microarray data for gene expression in silkworm larva (data unpublished), we analyzed the expression patterns of the eight common DEGs. Two of these genes were newly identified transcripts in RNA-seq; there is no corresponding microarray data. Microarray data of the remaining six genes were processed as previously reported (Xia et al. 2007; Zhang et al. 2012).

Authors' contributions Experimental concept and design: FD. Silkworm feeding: HH and DT. Research experimentation: XD and JL. Results and bioinformatics analysis: XD and SW. Manuscript writing: XD, XT, SW, $\mathrm{CL}$, and JS. All authors read and approved the final manuscript.
Funding information This work was supported by the National Natural Science Foundation of China [No. 31472153, No. 31830094]; the HiTech Research and Development 863 Program of China Grant [No. 2013AA102507]; and Funds of China Agriculture Research System [No. CARS-18-ZJ0102].

Availability of data and materials The datasets generated in this study are publicly available at NCBI-SRA (www.ncbi.nlm.nih.gov/sra) accession: SRR7894599, SRR7894600, SRR7894601, SRR7894602, SRR7894603, SRR7894604, SRR7894605, SRR7894606, SRR7894607, SRR7894608, SRR7894609, SRR7894610, SRR7894611, SRR7894612, SRR7894613, SRR7894614, SRR7894615, SRR7894616.

\section{Compliance with ethical standards}

Competing interests The authors declare that they have no competing interests.

Open Access This article is distributed under the terms of the Creative Commons Attribution 4.0 International License (http:// creativecommons.org/licenses/by/4.0/), which permits unrestricted use, distribution, and reproduction in any medium, provided you give appropriate credit to the original author(s) and the source, provide a link to the Creative Commons license, and indicate if changes were made.

\section{References}

Altschul SF, Madden TL, Schaffer AA, Zhang J, Zhang Z, Miller W, Lipman DJ (1997) Gapped BLAST and PSI-BLAST: a new generation of protein database search programs. Nucleic Acids Res 25: 3389-3402

Carballo GB, Honorato JR, de Lopes GPF, Spohr T (2018) A highlight on sonic hedgehog pathway. Cell Commun Signal 16:11. https://doi. org/10.1186/s12964-018-0220-7

Carroll SB, Gates J, Keys DN, Paddock SW, Panganiban GE, Selegue JE, Williams JA (1994) Pattern formation and eyespot determination in butterfly wings. Science 265:109-114

Daimon T et al (2010) The silkworm Green b locus encodes a quercetin 5O-glucosyltransferase that produces green cocoons with UVshielding properties. Proc Natl Acad Sci U S A 107:11471-11476. https://doi.org/10.1073/pnas.1000479107

Ewing BHL, Wendl MC, Green P (1998) Base-calling of automated sequencer traces using phred. I. Accuracy Assessment. Genome Res 8(3): 175-185

Florea L, Song L, Salzberg SL (2013) Thousands of exon skipping events differentiate among splicing patterns in sixteen human tissues. F1000Res 2:188. https://doi.org/10.12688/f1000research.2-188.v2

Futahashi R, Fujiwara H (2005) Melanin-synthesis enzymes coregulate stage-specific larval cuticular markings in the swallowtail butterfly, Papilio xuthus. Dev Genes Evol 215:519-529. https://doi.org/10. 1007/s00427-005-0014-y

Futahashi R, Banno Y, Fujiwara H (2010) Caterpillar color patterns are determined by a two-phase melanin gene prepatterning process: new evidence from tan and laccase2. Evol Dev 12:157-167. https://doi. org/10.1111/j.1525-142X.2010.00401.x

Futahashi R, Shirataki H, Narita T, Mita K, Fujiwara H (2012) Comprehensive microarray-based analysis for stage-specific larval camouflage pattern-associated genes in the swallowtail butterfly, Papilio xuthus. BMC Biol 10:46. https://doi.org/10.1186/17417007-10-46 
Gallant JR, Imhoff VE, Martin A, Savage WK, Chamberlain NL, Pote BL, Peterson C, Smith GE, Evans B, Reed RD, Kronforst MR, Mullen SP (2014) Ancient homology underlies adaptive mimetic diversity across butterflies. Nat Commun 5:4817. https://doi.org/ $10.1038 /$ ncomms 5817

He S, Tong X, Lu K, Lu Y, Luo J, Yang W, Chen M, Han MJ, Hu H, Lu C, Dai F (2016) Comparative analysis of transcriptomes among Bombyx mori strains and sexes reveals the genes regulating melanic morph and the related phenotypes. PLoS One 11:e0155061. https:// doi.org/10.1371/journal.pone.0155061

Keys DN et al (1999) Recruitment of a hedgehog regulatory circuit in butterfly eyespot evolution. Science 283:532-534

Kim D, Pertea G, Trapnell C, Pimentel H, Kelley R, Salzberg SL (2013) TopHat2: accurate alignment of transcriptomes in the presence of insertions, deletions and gene fusions. Genome Biol 14:R36. https:// doi.org/10.1186/gb-2013-14-4-r36

KonDo Y et al (2017) Toll ligand Spatzle3 controls melanization in the stripe pattern formation in caterpillars. Proc Natl Acad Sci U S A 114:8336-8341. https://doi.org/10.1073/pnas.1707896114

Koshikawa S, Giorgianni MW, Vaccaro K, Kassner VA, Yoder JH, Werner T, Carroll SB (2015) Gain of cis-regulatory activities underlies novel domains of wingless gene expression in Drosophila. Proc Natl Acad Sci U S A 112:7524-7529. https://doi.org/10.1073/pnas. 1509022112

Kronforst MR et al (2012) Unraveling the thread of nature's tapestry: the genetics of diversity and convergence in animal pigmentation. Pigment Cell Melanoma Res 25:411-433. https://doi.org/10.1111/ j.1755-148X.2012.01014.x

Langmead B, Trapnell C, Pop M, Salzberg SL (2009) Ultrafast and memoryefficient alignment of short DNA sequences to the human genome. Genome Biol 10:R25. https://doi.org/10.1186/gb-2009-10-3-r25

Linzen B (1974) The Tryptophan $\rightarrow$ omrnochrome pathway in insects 1 advances in Insect. Physiology 10:117-246

Livak KJ, Schmittgen TD (2001) Analysis of relative gene expression data using real-time quantitative PCR and the 2(-Delta Delta C(T)) Method. Methods 25:402-408. https://doi.org/10.1006/meth.2001.1262

Lu K et al (2018) qPrimerDB: a thermodynamics-based gene-specific qPCR primer database for 147 organisms. Nucleic Acids Res 46: D1229-D1236. https://doi.org/10.1093/nar/gkx725

Martin A, Reed RD (2014) Wnt signaling underlies evolution and development of the butterfly wing pattern symmetry systems. Dev Biol. https://doi.org/10.1016/j.ydbio.2014.08.031

Martin A et al (2012) Diversification of complex butterfly wing patterns by repeated regulatory evolution of a Wnt ligand. Proc Natl Acad Sci U S A 109:12632-12637. https://doi.org/10.1073/pnas.1204800109

Massey JH, Wittkopp PJ (2016) The genetic basis of pigmentation differences within and between Drosophila species. Curr Top Dev Biol 119:27-61. https://doi.org/10.1016/bs.ctdb.2016.03.004

Mazo-Vargas A et al (2017) Macroevolutionary shifts of WntA function potentiate butterfly wing-pattern diversity. Proc Natl Acad Sci U S A. https://doi.org/10.1073/pnas.1708149114

Nie H, Liu C, Cheng T, Li Q, Wu Y, Zhou M, Zhang Y, Xia Q (2014) Transcriptome analysis of integument differentially expressed genes in the pigment mutant (quail) during molting of silkworm, Bombyx mori. PLoS One 9:e94185. https://doi.org/10.1371/journal.pone.0094185

Nusslein-Volhard C, Wieschaus E (1980) Mutations affecting segment number and polarity in Drosophila. Nature 287:795-801

Osanai-Futahashi M et al (2016) Positional cloning of a Bombyx pinkeyed white egg locus reveals the major role of cardinal in ommochrome synthesis. Heredity 116:135-145. https://doi.org/10. 1038/hdy.2015.74

Roberts A, Pimentel H, Trapnell C, Pachter L (2011) Identification of novel transcripts in annotated genomes using RNA-Seq. Bioinformatics 27: 2325-2329. https://doi.org/10.1093/bioinformatics/btr355

Robinson MD, McCarthy DJ, Smyth GK (2010) edgeR: a Bioconductor package for differential expression analysis of digital gene expression data. Bioinformatics 26:139-140. https://doi.org/10. 1093/bioinformatics/btp616

Shirataki H, Futahashi R, Fujiwara H (2010) Species-specific coordinated gene expression and trans-regulation of larval color pattern in three swallowtail butterflies. Evol Dev 12:305-314. https://doi.org/10. 1111/j.1525-142X.2010.00416.x

Swarup S, Pradhan-Sundd T, Verheyen EM (2015) Genome-wide identification of phospho-regulators of Wnt signaling in Drosophila. Development 142:1502-1515. https://doi.org/10.1242/dev.116715

Takahashi A (2013) Pigmentation and behavior: potential association through pleiotropic genes in Drosophila. Genes Genet Syst 88:165-174

Toku AE, Tekir SD, Ozbayraktar FB, Ulgen KO (2011) Reconstruction and crosstalk of protein-protein interaction networks of Wnt and Hedgehog signaling in Drosophila melanogaster. Comput Biol Chem 35:282292. https://doi.org/10.1016/j.compbiolchem.2011.07.002

Tong X, Lindemann A, Monteiro A (2012) Differential involvement of Hedgehog signaling in butterfly wing and eyespot development. PLoS One 7:e51087. https://doi.org/10.1371/journal.pone.0051087

Tong X, Hrycaj S, Podlaha O, Popadic A, Monteiro A (2014) Overexpression of Ultrabithorax alters embryonic body plan and wing patterns in the butterfly Bicyclus anynana. Dev Biol 394:357-366. https://doi.org/10.1016/j.ydbio.2014.08.020

Wang P, Qiu Z, Xia D, Tang S, Shen X, Zhao Q (2017) Transcriptome analysis of the epidermis of the purple quail-like (q-lp) mutant of silkworm, Bombyx mori. PLoS One 12:e175994. https://doi.org/ 10.1371/journal.pone.0175994

Wittkopp PJ, Beldade P (2009) Development and evolution of insect pigmentation: genetic mechanisms and the potential consequences of pleiotropy. Semin Cell Dev Biol 20:65-71. https://doi.org/10. 1016/j.semcdb.2008.10.002

Wittkopp PJ, Carroll SB, Kopp A (2003) Evolution in black and white: genetic control of pigment patterns in Drosophila. Trends Genet 19: 495-504. https://doi.org/10.1016/S0168-9525(03)00194-X

Wu S, Tong X, Peng C, Xiong G, Lu K, hu H, Tan D, Li C, Han M, Lu C, Dai $F$ (2016) Comparative analysis of the integument transcriptomes of the black dilute mutant and the wild-type silkworm Bombyx mori. Sci Rep 6:26114. https://doi.org/10.1038/srep26114

Xia Q, Cheng D, Duan J, Wang G, Cheng T, Zha X, Liu C, Zhao P, Dai F, Zhang Z, He N, Zhang L, Xiang Z (2007) Microarray-based gene expression profiles in multiple tissues of the domesticated silkworm, Bombyx mori. Genome Biol 8:R162. https://doi.org/10.1186/gb2007-8-8-r162

Xiong $\mathrm{G}$ et al (2017) Body shape and coloration of silkworm larvae are influenced by a novel cuticular protein. Genetics. https://doi.org/10. 1534/genetics.117.300300

Yamaguchi J, Banno Y, Mita K, Yamamoto K, Ando T, Fujiwara H (2013) Periodic Wnt1 expression in response to ecdysteroid generates twin-spot markings on caterpillars. Nat Commun 4:1857. https://doi.org/10.1038/ncomms2778

Yoda S, Yamaguchi J, Mita K, Yamamoto K, Banno Y, Ando T, Daimon T, Fujiwara H (2014) The transcription factor Apontic-like controls diverse colouration pattern in caterpillars. Nat Commun 5:4936. https://doi.org/10.1038/ncomms5936

Zhang Y, Dong Z, Liu S, Yang Q, Zhao P, Xia Q (2012) Identification of novel members reveals the structural and functional divergence of lepidopteran-specific Lipoprotein 11 family. Funct Integr Genomics 12:705-715. https://doi.org/10.1007/s10142-012-0281-4

Zhang L, Martin A, Perry MW, van der Burg KR, Matsuoka Y, Monteiro A, Reed RD (2017) Genetic basis of melanin pigmentation in butterfly wings. Genetics 205:1537-1550. https://doi.org/10.1534/ genetics.116.196451

Publisher's note Springer Nature remains neutral with regard to jurisdictional claims in published maps and institutional affiliations. 